\title{
Efforts to Develop KRAS Inhibitors
}

\author{
Matthew Holderfield \\ NCl-Ras Initiative, Cancer Research Technology Program, Frederick National Laboratory for Cancer Research, \\ Leidos Biomedical Research, Frederick, Maryland 21702 \\ Correspondence: matthew.holderfield@nih.gov
}

The high prevalence of KRAS mutations in human cancers and the lack of effective treatments for patients ranks KRAS among the most highly sought-after targets for preclinical oncologists. Pharmaceutical companies and academic laboratories have tried for decades to identify small molecule inhibitors of oncogenic KRAS proteins, but little progress has been made and many have labeled KRAS undruggable. However, recent progress in in silico screening, fragmentbased drug design, disulfide tethered screening, and some emerging themes in RAS biology have caused the field to reconsider previously held notions about targeting KRAS. This review will cover some of the historical efforts to identify RAS inhibitors, and some of the most promising efforts currently being pursued.

$T_{\mathrm{p}}^{\mathrm{h}}$ he age of whole-genome sequencing has paved the road for "personalized" medicine in oncology. The Cancer Genome Atlas Research Network (2017), the Cancer Cell Line Encyclopedia (Barretina et al. 2012), the Genomics of Drug Sensitivity in Cancer (Yang et al. 2013), and similar efforts aim to catalog driver mutations with actionable and effective treatment regimens. While significant progress has been made in some notable areas, including BRAF-mutated melanomas (Davies et al. 2002; Chapman et al. 2011) and BRCA-mutated breast and ovarian cancers (Fong et al. 2009; The Cancer Genome Atlas Research Network 2011), many of the most common oncogenes were identified decades ago and remain the most deadly and elusive oncology drug targets. Perhaps chief among these is KRAS.

Interest in KRAS as a cancer target stems from work with rodent sarcoma viruses in the late 1970s. First, DNAs from viruses and later from human tumor cells were shown to be sufficient to transform NIH-3T3 cells in culture (Shih et al. 1979; Shih et al. 1981). It was not until the early 1980s that KRAS was identified as the causative oncogene (Der et al. 1982; Parada and Weinberg 1983). By 1987, KRAS oncogenes were cloned from multiple tumor biopsies of lung, colon, and pancreas cancers (Santos et al. 1984; Bos et al. 1987; Forrester et al. 1987; Rodenhuis et al. 1987; Almoguera et al. 1988). By this time, all three RAS isoforms, $\mathrm{H}-, \mathrm{N}-$, and $\mathrm{K}$ RAS were known and mutations at codons 12, 13, and 61 of a RAS isoform were shown to be sufficient to induce cell-cycle progression. Finally, the HRAS crystal structure revealed that the cancerassociated mutations at codons G12, G13, and Q61 are each located at a site of guanosine triphosphate (GTP) binding, suggesting a common mechanism of oncogenesis (Pai et al. 1989).

Editors: Linda VanAelst, Julian Downward, and Frank McCormick

Additional Perspectives on Ras and Cancer in the 21st Century available at www.perspectivesinmedicine.org

Copyright (C 2018 Cold Spring Harbor Laboratory Press; all rights reserved; doi: 10.1101/cshperspect.a031864 Cite this article as Cold Spring Harb Perspect Med 2018;8:a031864 
M. Holderfield

It was later determined that RAS proteins are prenylated small GTPases. $\mathrm{H}$-, N-, and $\mathrm{K}$ RAS are all farnesylated at the carboxyl terminus. $\mathrm{H}$ - and N-RAS as well as one of the two KRAS splice variants (KRAS 4A) are also palmitoylated, whereas KRAS 4B contains a series of charged lysine residues near the carboxyl terminus that aid in membrane localization (Hancock 2003). Fully processed RAS proteins localize to the plasma membrane where they are activated upon growth factor stimulus. Guanosine exchange factors (GEFs) promote RAS-GTP association to offset the high rate of GTP hydrolysis in cells. Hydrolysis is stimulated by GTPase-activating proteins (GAPs) (Trahey and McCormick 1987). Among the most well-characterized RAS GAP proteins are p120 and NF1, which increase GTP hydrolysis $>10,000$-fold over the intrinsic rate. GAP proteins all contain a catalytic arginine residue that fits into the active site of RAS to stimulate hydrolysis of the terminal GTP phosphate. Mutations at RAS G12, G13, or Q61 prevent the GAP arginine from accessing GTP, which stabilizes RAS in the GTP-bound state (Scheffzek et al. 1997).

In the GTP-bound form, RAS proteins associate with and activate a variety of effector molecules including phosphatidylinositol-3 kinase (PI3K) and the RAF kinases. RAF kinases activate the mitogen-activated protein kinase (MAPK) pathway by phosphorylating MEK1 and MEK2, which in turn phosphorylate ERK1 and ERK2, which phosphorylate and activate many transcription factors that promote cellcycle progression. Alternatively, PI3K activates $\mathrm{AKT}$ and mechanistic target of rapamycin (mTOR) signaling to promote protein translation. Unlike RAF kinases, PI3K can be activated independent of RAS by receptor tyrosine kinases (RTKs) or indirectly through G-protein-coupled receptor (GPCR) and integrin signaling, but both effector pathways are strongly implicated in cancer progression and have been evaluated as therapeutic targets for KRAS-mutated cancers. Genetically engineered mouse models demonstrate that KRAS G12D-driven nonsmall-cell lung cancer (NSCLC) requires a single RAS isoform, CRAF, as well as the RAF client proteins, MEK1/MEK2, and the MEK sub- strates, ERK1/ERK2 (Blasco et al. 2011). Likewise, KRAS activation of PI3K is also essential in a similar NSCLC mouse model (Castellano et al. 2013). Unfortunately, KRAS-mutated cancers and many of the available cell lines have proven unresponsive to MEK, RAF, or PI3K inhibitors administered as single agents (Barretina et al. 2012). Therefore, cotargeting the MAPK and PI3K pathways may be required to treat KRASdriven cancers (Engelman et al. 2008). Unfortunately, coadministration of PI3K and MEK inhibitors is not well tolerated. Phase I clinical trials reported grade 3 and 4 dose-limiting toxicities, which is thought to limit clinical efficacy (Bedard et al. 2015). Unless dosing regimens can be modified to reduce general toxicity, cotargeting MAPK and PI3K does not appear to be a viable therapeutic option, which further illustrates the unmet clinical need for effective treatment strategies for KRAS-mutated cancers. This has, in part, nucleated a resurgence for basic science and drug discovery efforts to target oncogenic KRAS proteins and to identify new targets required for KRAS-driven oncogenesis.

\section{DIRECT TARGETING OF KRAS PROTEINS}

The difficulties drugging RAS stem from the small size of the protein, just $21 \mathrm{kD}$, with a lack of an apparent "pocket" for a small molecule to bind apart from the nucleotide substratebinding site. However, the high picomolar affinity reported for nucleotide substrates paired with the high millimolar cellular concentration of GTP suggests that this strategy is thermodynamically and pharmacologically unattainable. As such, RAS proteins are commonly referred to as "undruggable," but a renewed interest in targeting KRAS may change this paradigm (Stephen et al. 2014). This review will focus on many of the strategies to target KRAS in the past, as well as some promising efforts that are currently being pursued.

\section{Targeting Nucleotide Exchange}

SCH54292 was described in the first report of a small molecule that binds directly to RAS (Taveras et al. 1997). The series was developed from 
a biochemical screen designed to identify inhibitors of nucleotide exchange, and nuclear magnetic resonance (NMR) studies showed that it interacts with the Switch II domain of RAS in the guanosine diphosphate (GDP)-bound conformation. Similar studies have since been performed by other groups using different nucleotide exchange assays, but all nucleotide exchange inhibitors typically suffer from low affinity for RAS (Cox et al. 2014) and often lack activity in cells. None of the compounds identified have been developed for clinical use.

More recently, three groups have used NMR to identify small molecule fragments that bind to RAS (Maurer et al. 2012; Sun et al. 2014; Winter et al. 2015). This strategy seeks to identify novel compound-binding pockets using low molecular weight chemical matter and to improve affinity by increasing the inhibitor size through a rational structure-based drug design or by chemically linking multiple fragments with low affinity for the target protein. Many of the chemical fragments identified bind to RAS in a shallow pocket near the $\alpha 2$ helix, which is also the site for SOS binding (Boriack-Sjodin et al. 1998). Indeed, the fragments inhibit SOS binding and SOS-dependent nucleotide exchange in vitro. In theory, this method could be used to develop a small molecule with high enough affinity for RAS to inhibit activity in cells. The $\alpha 2$ compound binding site is not apparent in the apocrystal structures, and it is not clear to what extent the pocket can be exploited with a larger compound. It is also not clear whether such a strategy will have activity in KRAS-mutated cells because KRAS oncoproteins are not affected by epidermal growth factor receptor (EGFR) or other RTK inhibitors (Khambata-Ford et al. 2007) and are presumably not responsive to upstream perturbations, including inhibition of SOS activity.

\section{Targeting RAS-Effector Interactions}

Perhaps a more direct mechanism of action for a RAS inhibitor might be to inhibit RAS-effector interactions. Protein-protein interactions are not always easy to target as proteins often interact through multiple residues along flat surfaces, which makes inhibition thermodynamically unfavorable with a small molecule. However, a single-point mutation in the RAS-binding domain of RAF kinase or RAL-GDS is sufficient to abolish RAS-RAF or RAS-RAL-GDS binding (Fabian et al. 1994), suggesting that even a minor disruption of the RAS-effector binding site may prevent binding. Thus, assays that measure KRAS-effector interactions in vitro and in cell systems have been used to screen many compound libraries. One such compound, MCP1, was identified in an HRAS-CRAF yeast two-hybrid screen, and demonstrated activity in NRASmutated HT1080, KRAS-mutated PANC-1, and A549 cells in the 10 - to $20-\mu \mathrm{M}$ range, but no inhibition of BRAF V600E-mutated A2058 cells was observed (Kato-Stankiewicz et al. 2002). It is not clear whether MCP1 binds directly to RAS or CRAF, and the compound series is not potent enough for therapeutic activity. However, the proposed mechanism of action remains a viable approach for inhibiting oncogenic KRAS signal transduction.

Peptide chemistries are often used to increase compound library diversity and they are well suited to target protein-protein interactions. A large combinatorial library of cyclic peptides was screened for the ability to inhibit KRAS-RAF1 binding in vitro. The cyclic peptide, cyclorasin, was shown to bind RAS only in the GTP-bound state and compete directly with RAS effectors (Wu et al. 2013). The peptide was further optimized for cell permeability, and the authors report submicromolar IC50s in KRASmutated cancer cell lines (Trinh et al. 2016). However, the large molecular weight of the compounds may present significant pharmacokinetic challenges for clinical development.

\section{In Silico Screening}

Computer "docking" studies offer a cost-effective alternative to wet-bench screening efforts to identify potential chemical leads for difficult targets. Computational methods use structural information obtained from crystallography and NMR to virtually dock small molecules into putative binding pockets. Molecules predicted to dock are then synthesized and tested in func- 
M. Holderfield

tional biological assays. Although in silico docking does not always correlate perfectly with biological activity, in silico screens can start with a large virtual library and enrich for compounds likely to bind to the target of interest. The library size is limited only by computing time needed for the docking experiments. Virtual screening methods can accommodate libraries orders of magnitude larger than what can be screened in biochemical assays. This allows for a greater diversity of chemical space that can be tested and reduces the size of the library to be tested in subsequent biochemical or biophysical experiments.

The first-reported effort to target the effector-binding interface on RAS revealed Kobe 2602, which inhibited SOS-mediated exchange in vitro and inhibited RAS signal transduction pathways and proliferation of HRAS G12Vtransformed NIH-3T3 cells (Shima et al. 2013). The compound also demonstrated modest activity in a xenograft model implanted with KRAS G12V SW480 cells. Whereas the effort demonstrated proof-of-concept for an in silico-based screening approach, the 2602 chemical series itself has not been pursued for further development because of instability and general toxicity.

Improved methods and increased computing power continue to advance in silico drugscreening efforts. A recent publication sought to target KRAS-RAF1 interactions using iterative in silico docking to develop 3144, a compound with activity on KRAS-mutated cancer cell lines in the 1- to 10- $\mu \mathrm{M}$ range (Shima et al. 2013). The authors went on to validate the in silico docking to KRAS by microscale thermophoresis, isothermal calorimetry, and NMR, and reported a dissociation constant also in the low micromolar range. Although not yet validated by crystallography, the NMR structure confirmed the binding site to be directly on the Switch I and II loops, and the binding is predicted to be competitive with effector proteins. Perhaps most impressively, 3144 had a dramatic response in KRAS-mutated cell lines and patient-derived xenograft mouse models. With a molecular weight of $717.66,3144$ is already quite large for a small molecule, and it is not yet clear whether the series can be further developed to improve potency for clinical development.

\section{Disulfide Tethering}

A variation of fragment-based screening was recently used to identify a previously unrecognized small molecule-binding pocket on KRAS. Disulfide fragment libraries were designed to identify low-affinity small-molecule fragments that bind with low affinity to shallow pockets and react with nearby cysteine residues. The small molecule-protein complex can then be detected by mass spectrometry to identify hit compounds. Shokat and colleagues used such a library to identify fragments that bind to the oncogenic KRAS G12C protein in a pocket adjacent to the nucleotide-binding site within the Switch II region (Ostrem et al. 2013). This pocket was not apparent in the apocrystal structures, but appears to be induced or stabilized by the compound. The disulfide reactive groups were then converted to electrophilic acrylamides or vinyl sulfonamides, which irreversibly attach to cysteine residues in the reducing environment of a cell. The binding pocket was subsequently expanded to accommodate larger small molecules with higher affinity and the current generation of inhibitors is active in cells in the nanomolar range (Lito et al. 2016; Patricelli et al. 2016). Furthermore, by targeting the oncogenic mutation itself (which happens to be a cysteine), the compounds potentially will benefit from an extraordinary therapeutic index because the compounds will only react with the KRAS G12C protein, but not the wild-type RAS molecules that harbor a glycine at codon 12 .

\section{INHIBITING MEMBRANE LOCALIZATION}

In addition to directly targeting KRAS proteins, several efforts have been made to target KRASdriven cancers by inhibiting cellular processes required for KRAS function. One of the first and most extensive efforts was to inhibit farnesyl transferase, which is required for $\mathrm{H}-\mathrm{N}$ - and KRAS farnesylation (End et al. 2001). The small molecule, Tipifarnib was developed and tested in clinical trials for this purpose. Although the 
compounds were quite effective at inhibiting farnesyl transferase, it was discovered that KRAS itself does not require farnesylation for membrane localization. Although HRAS-dependent cells are quite sensitive to farnesyl transferase inhibitors, geranylgeranyl transferase prenylates the carboxyl terminus of KRAS (but not HRAS) upon farnesyl transferase inhibition, which restores membrane localization and full biochemical activity in the presence of Tipifarnib. Unfortunately, a dual inhibitor of farnesyl transferase and geranylgeranyl transferase reached dose-limiting toxicity but did not inhibit KRAS prenylation in human patients (Lobell et al. 2002). Although farnesyl transferase inhibitors are not effective in KRAS-mutated cancers, they are currently being evaluated for use in HRAS-mutated cancers, which represent $5 \%$ of bladder and thyroid cancers.

\section{ICMT and RCE1}

After prenylation, the final posttranslational modifications before KRAS is transported to the membrane are proteolysis of the three carboxy-terminal resides and then methylation of the prenylated cysteine. These are done by RCE1 (Ras converting CAAX endopeptidase 1) (Boyartchuk et al. 1997) and ICMT (isoprenylcysteine carboxyl methyltransferase) (Bergo et al. 2000). Inhibitors of both enzymes have been developed and are reported to mislocalize RAS molecules in cells, but RCE1 inhibitors have not been fully evaluated in vivo, and the antiproliferative effects of ICMT inhibition are not RAS specific (Bergo et al. 2004), suggesting that ICMT may be required for normal cellular function in KRAS wild-type cells.

\section{PDE6-Delta}

It was discovered that the KRAS splice forms $4 \mathrm{~A}$ and $4 \mathrm{~B}$, which differ in the last 25 amino acids, are transported throughout the cell by different mechanisms (Wright and Philips 2006), and that KRAS 4B may require a chaperone, PDE6d (Chandra et al. 2011; Schmick et al. 2014). PDE6d is a prenyl-binding protein that is thought to solubilize prenylated proteins by shielding the hydrophobic farnesyl chain when KRAS is not bound to a membrane surface (Dharmaiah et al. 2016). A small molecule inhibitor, deltarasin, was developed to prevent PDE6d from binding to KRAS by occupying the prenyl-binding pocket on PDE6d (Zimmermann et al. 2013). Cells treated with the compound cause a mislocalization of KRAS $4 \mathrm{~B}$ proteins, which accumulate on endomembranes. Cancer cells are also quite sensitive to deltarasin, though it is unclear whether KRAS mislocalization inhibits oncogenic KRAS 4B signal transduction or whether cellular toxicity is a result of inhibition of another PDE6d substrate. A second generation of PDE6d inhibitors was subsequently published with improved selectivity (Papke et al. 2016). Further investigation into PDE6d and similar mechanisms that regulate KRAS localization is likely to reveal additional cancer-relevant biology that can be exploited for therapeutic intervention.

\section{DIMERIZATION AND MULTIMERIZATION}

One emerging area of interest is the idea that activated RAS molecules may dimerize at the plasma membrane. Because RAF activation is thought to require dimerization, RAS dimerization would be a logical prerequisite, and there is some evidence that RAS molecules form highorder complexes. Superresolution single-molecule imaging studies of PA-mCherry-tagged KRAS proteins expressed in mammalian cells revealed the presences of apparent KRAS dimers (Nan et al. 2015). Artificially induced dimerization of KRAS molecules also activated the MAPK pathway, suggesting that KRAS dimerization is sufficient for signal transduction, yet it remains unclear whether RAS dimerization is required for oncogenic or wild-type KRAS signal transduction. One study of purified HRAS proteins in supported lipid bilayers identified a putative dimerization interface and a point mutation at Y64C that abolished dimerization (Lin et al. 2014). However, it is dimerization in cells that appears to be more complicated. The proposed dimerization interface is also within the effector-binding site and Y64 is required for binding to the RAS GEF, SOS. Other studies 
M. Holderfield

suggest that RAS may form higher-order "nanoclusters" in cells that are associated with and may be required for signal transduction (Zhou and Hancock 2015), and cellular expression of a "monobody" protein engineered to bind directly to RAS was shown to disrupt RAS nanoclusters and perturb signal transduction (Spencer-Smith et al. 2017). These studies offer some optimism that disrupting RAS-RAS interactions could be a viable therapeutic mechanism of action, but the underlying biology is not fully understood.

\section{CONCLUDING REMARKS}

Over 35 years of investigation has yet to produce an effective treatment for the large patient population with KRAS-mutated cancers. All the essential signal transduction pathways feeding into and emanating from KRAS, including multiple RTKs, RAF, MEK, PI3K, AKT, mTOR, and a host of ancillary proteins, have all been successfully targeted with small molecules. Inhibitors have been evaluated in cancer patients, and many have been approved by regulatory bodies, yet none of the treatments have proven effective in KRAS-mutated cancers. It is therefore understandable why KRAS is frequently referred to as one of the "undruggable" oncology targets. However, recent advances in in silico docking, covalent tethering, and fragment-based drug screening offer new hope for developing direct inhibitors of RAS proteins. These and other technologies are likely to yield small molecules that bind to KRAS with sufficient affinity for activity in cells and whole animals. Additionally, new biology has shed some light onto mechanisms required for RAS trafficking, membrane localization, and membrane dynamics, which may reveal new targets or targetable mechanisms.

\section{ACKNOWLEDGMENTS}

This project is funded in part with federal funds from the National Cancer Institute, National Institutes of Health (NIH), under Contract No. HHSN261200800001E. The content of this publication does not necessarily reflect the views or policies of the Department of Health and Human Services, and the mention of trade names, commercial products, or organizations does not imply endorsement by the U.S. Government.

\section{REFERENCES}

Barretina J, Caponigro G, Stransky N, Venkatesan K, Margolin AA, Kim S, Wilson CJ, Lehar J, Kryukov GV, Sonkin D, et al. 2012. The Cancer Cell Line Encyclopedia enables predictive modelling of anticancer drug sensitivity. $\mathrm{Na}$ ture 483: 603-607.

Bedard PL, Tabernero J, Janku F, Wainberg ZA, Paz-Ares L, Vansteenkiste J, Van Cutsem E, Perez-Garcia J, Stathis A, Britten CD, et al. 2015. A phase Ib dose-escalation study of the oral pan-PI3K inhibitor buparlisib (BKM120) in combination with the oral MEK1/2 inhibitor trametinib (GSK1120212) in patients with selected advanced solid tumors. Clin Cancer Res 21: 730-738.

Almoguera C, Shibata D, Forrester K, Martin J, Arnheim N, Perucho M. 1988. Most human carcinomas of the exocrine pancreas contain mutant c-K-ras genes. Cell 53: 549-554.

Bergo MO, Leung GK, Ambroziak P, Otto JC, Casey PJ, Young SG. 2000. Targeted inactivation of the isoprenylcysteine carboxyl methyltransferase gene causes mislocalization of K-Ras in mammalian cells. J Biol Chem 275: 17605-17610.

Bergo MO, Gavino BJ, Hong C, Beigneux AP, McMahon M, Casey PJ, Young SG. 2004. Inactivation of Icmt inhibits transformation by oncogenic K-Ras and B-Raf. J Clin Invest 113: 539-550.

Blasco RB, Francoz S, Santamaria D, Canamero M, Dubus P, Charron J, Baccarini M, Barbacid M. 2011. c-Raf, but not B-Raf, is essential for development of K-Ras oncogenedriven non-small-cell lung carcinoma. Cancer Cell 19: 652-663.

Boriack-Sjodin PA, Margarit SM, Bar-Sagi D, Kuriyan J. 1998. The structural basis of the activation of Ras by Sos. Nature 394: 337-343.

Bos JL, Fearon ER, Hamilton SR, Verlaan-de Vries M, van Boom JH, van der Eb AJ, Vogelstein B. 1987. Prevalence of ras gene mutations in human colorectal cancers. $\mathrm{Na}$ ture 327: 293-297.

Boyartchuk VL, Ashby MN, Rine J. 1997. Modulation of Ras and a-factor function by carboxyl-terminal proteolysis. Science 275: 1796-1800.

Castellano E, Sheridan C, Thin MZ, Nye E, Spencer-Dene B, Diefenbacher ME, Moore C, Kumar MS, Murillo MM, Gronroos E, et al. 2013. Requirement for interaction of PI3-kinase $\mathrm{p} 110 \alpha$ with RAS in lung tumor maintenance. Cancer Cell 24: 617-630.

Chandra A, Grecco HE, Pisupati V, Perera D, Cassidy L, Skoulidis F, Ismail SA, Hedberg C, Hanzal-Bayer M, Venkitaraman AR, et al. 2011. The GDI-like solubilizing factor PDE $\delta$ sustains the spatial organization and signalling of Ras family proteins. Nat Cell Biol 14: 148-158.

Chapman PB, Hauschild A, Robert C, Haanen JB, Ascierto P, Larkin J, Dummer R, Garbe C, Testori A, Maio M, et al. 2011. Improved survival with vemurafenib in melanoma with BRAF V600E mutation. N Engl J Med 364: 2507 2516. 
Cox AD, Fesik SW, Kimmelman AC, Luo J, Der CJ. 2014 Drugging the undruggable RAS: Mission possible? Nat Rev Drug Discov 13: 828-851.

Davies H, Bignell GR, Cox C, Stephens P, Edkins S, Clegg S, Teague J, Woffendin H, Garnett MJ, Bottomley W, et al. 2002. Mutations of the BRAF gene in human cancer. Nature 417: 949-954.

Der CJ, Krontiris TG, Cooper GM. 1982. Transforming genes of human bladder and lung carcinoma cell lines are homologous to the ras genes of Harvey and Kirsten sarcoma viruses. Proc Natl Acad Sci 79: 3637-3640.

Dharmaiah S, Bindu L, Tran TH, Gillette WK, Frank PH, Ghirlando R, Nissley DV, Esposito D, McCormick F, Stephen AG, et al. 2016. Structural basis of recognition of farnesylated and methylated KRAS4b by PDES. Proc Natl Acad Sci 113: E6766-E6775.

End DW, Smets G, Todd AV, Applegate TL, Fuery CJ, Angibaud P, Venet M, Sanz G, Poignet H, Skrzat S, et al 2001. Characterization of the antitumor effects of the selective farnesyl protein transferase inhibitor R115777 in vivo and in vitro. Cancer Res 61: 131-137.

Engelman JA, Chen L, Tan X, Crosby K, Guimaraes AR, Upadhyay R, Maira M, McNamara K, Perera SA, Song Y, et al. 2008. Effective use of PI3K and MEK inhibitors to treat mutant Kras G12D and PIK3CA H1047R murine lung cancers. Nat Med 14: 1351-1356.

Fabian JR, Vojtek AB, Cooper JA, Morrison DK. 1994. A single amino acid change in Raf-1 inhibits Ras binding and alters Raf-1 function. Proc Natl Acad Sci 91: 59825986.

Fong PC, Boss DS, Yap TA, Tutt A, Wu P, Mergui-Roelvink M, Mortimer P, Swaisland H, Lau A, O'Connor MJ, et al 2009. Inhibition of poly(ADP-ribose) polymerase in tumors from BRCA mutation carriers. N Engl J Med 361: $123-134$

Forrester K, Almoguera C, Han K, Grizzle WE, Perucho M. 1987. Detection of high incidence of K-ras oncogenes during human colon tumorigenesis. Nature 327: 298 303.

Hancock JF. 2003. Ras proteins: Different signals from different locations. Nat Rev Mol Cell Biol 4: 373-384.

Kato-Stankiewicz J, Hakimi I, Zhi G, Zhang J, Serebriiskii I, Guo L, Edamatsu H, Koide H, Menon S, Eckl R, et al. 2002. Inhibitors of Ras/Raf-1 interaction identified by two-hybrid screening revert Ras-dependent transformation phenotypes in human cancer cells. Proc Natl Acad Sci 99: 14398-14403.

Khambata-Ford S, Garrett CR, Meropol NJ, Basik M, Harbison CT, Wu S, Wong TW, Huang X, Takimoto $\mathrm{CH}$ Godwin AK, et al. 2007. Expression of epiregulin and amphiregulin and K-ras mutation status predict disease control in metastatic colorectal cancer patients treated with cetuximab. J Clin Oncol 25: 3230-3237.

Lin WC, Iversen L, Tu HL, Rhodes C, Christensen SM, Iwig JS, Hansen SD, Huang WY, Groves JT. 2014. H-Ras forms dimers on membrane surfaces via a protein-protein interface. Proc Natl Acad Sci 111: 2996-3001.

Lito P, Solomon M, Li LS, Hansen R, Rosen N. 2016. Allelespecific inhibitors inactivate mutant KRAS G12C by a trapping mechanism. Science 351: 604-608.

Lobell RB, Liu D, Buser CA, Davide JP, DePuy E, Hamilton K, Koblan KS, Lee Y, Mosser S, Motzel SL, et al. 2002.
Preclinical and clinical pharmacodynamic assessment of L-778,123, a dual inhibitor of farnesyl:protein transferase and geranylgeranyl:protein transferase type-I. $\mathrm{Mol} \mathrm{Can-}$ cer Ther 1: 747-758.

Maurer T, Garrenton LS, Oh A, Pitts K, Anderson DJ, Skelton NJ, Fauber BP, Pan B, Malek S, Stokoe D, et al. 2012. Small-molecule ligands bind to a distinct pocket in Ras and inhibit SOS-mediated nucleotide exchange activity. Proc Natl Acad Sci 109: 5299-5304.

Nan X, Tamguney TM, Collisson EA, Lin LJ, Pitt C, Galeas J, Lewis S, Gray JW, McCormick F, Chu S. 2015. Ras-GTP dimers activate the mitogen-activated protein kinase (MAPK) pathway. Proc Natl Acad Sci 112: 7996-8001.

Ostrem JM, Peters U, Sos ML, Wells JA, Shokat KM. 2013. KRas $^{\mathrm{G} 12 \mathrm{C}}$ inhibitors allosterically control GTP affinity and effector interactions. Nature 503: 548-551.

Pai EF, Kabsch W, Krengel U, Holmes KC, John J, Wittinghofer A. 1989. Structure of the guanine-nucleotidebinding domain of the Ha-ras oncogene product p21 in the triphosphate conformation. Nature 341: 209-214.

Papke B, Murarka S, Vogel HA, Martin-Gago P, Kovacevic M, Truxius DC, Fansa EK, Ismail S, Zimmermann G Heinelt K, et al. 2016. Identification of pyrazolopyridazinones as PDE $\delta$ inhibitors. Nat Commun 7: 11360

Parada LF, Weinberg RA. 1983. Presence of a Kirsten murine sarcoma virus ras oncogene in cells transformed by 3 methylcholanthrene. Mol Cell Biol 3: 2298-2301.

Patricelli MP, Janes MR, Li LS, Hansen R, Peters U, Kessler LV, Chen Y, Kucharski JM, Feng J, Ely T, et al. 2016 Selective inhibition of oncogenic KRAS output with small molecules targeting the inactive state. Cancer Discov 6: 316-329.

Rodenhuis S, van de Wetering ML, Mooi WJ, Evers SG, van Zandwijk N, Bos JL. 1987. Mutational activation of the Kras oncogene. A possible pathogenetic factor in adenocarcinoma of the lung. N Engl J Med 317: 929-935.

Santos E, Martin-Zanca D, Reddy EP, Pierotti MA, Della Porta G, Barbacid M. 1984. Malignant activation of a Kras oncogene in lung carcinoma but not in normal tissue of the same patient. Science 223: 661-664.

Scheffzek K, Ahmadian MR, Kabsch W, Wiesmuller L, Lautwein A, Schmitz F, Wittinghofer A. 1997. The Ras-RasGAP complex: Structural basis for GTPase activation and its loss in oncogenic Ras mutants. Science 277: 333-338.

Schmick M, Vartak N, Papke B, Kovacevic M, Truxius DC, Rossmannek L, Bastiaens PIH. 2014. KRas localizes to the plasma membrane by spatial cycles of solubilization, trapping and vesicular transport. Cell 157: 459-471.

Shih C, Shilo BZ, Goldfarb MP, Dannenberg A, Weinberg RA. 1979. Passage of phenotypes of chemically transformed cells via transfection of DNA and chromatin. Proc Natl Acad Sci 76: 5714-5718.

Shih C, Padhy LC, Murray M, Weinberg RA. 1981. Transforming genes of carcinomas and neuroblastomas introduced into mouse fibroblasts. Nature 290: 261-264.

Shima F, Yoshikawa Y, Ye M, Araki M, Matsumoto S, Liao J, Hu L, Sugimoto T, Ijiri Y, Takeda A, et al. 2013. In silico discovery of small-molecule Ras inhibitors that display antitumor activity by blocking the Ras-effector interaction. Proc Natl Acad Sci 110: 8182-8187. 
M. Holderfield

Spencer-Smith R, Koide A, Zhou Y, Eguchi RR, Sha F, Gajwani P, Santana D, Gupta A, Jacobs M, Herrero-Garcia E, et al. 2017. Inhibition of RAS function through targeting an allosteric regulatory site. Nat Chem Biol 13: 62-68.

Stephen AG, Esposito D, Bagni RK, McCormick F. 2014 Dragging Ras back in the ring. Cancer Cell 25: 272-281.

Sun Q, Phan J, Friberg AR, Camper DV, Olejniczak ET, Fesik SW. 2014. A method for the second-site screening of KRas in the presence of a covalently attached first-site ligand. J Biomol NMR 60: 11-14.

Taveras AG, Remiszewski SW, Doll RJ, Cesarz D, Huang EC, Kirschmeier P, Pramanik BN, Snow ME, Wang YS, del Rosario JD, et al. 1997. Ras oncoprotein inhibitors: The discovery of potent, ras nucleotide exchange inhibitors and the structural determination of a drug-protein complex. Bioorg Med Chem 5: 125-133.

The Cancer Genome Atlas Research Network. 2011. Integrated genomic analyses of ovarian carcinoma. Nature 474: 609-615.

The Cancer Genome Atlas Research Network. 2017. National Cancer Institute and the National Human Genome Research Institute, National Institutes of Health, Bethesda, MD.

Trahey M, McCormick F. 1987. A cytoplasmic protein stimulates normal N-ras p21 GTPase, but does not affect oncogenic mutants. Science 238: 542-545.

Trinh TB, Upadhyaya P, Qian Z, Pei D. 2016. Discovery of a direct ras inhibitor by screening a combinatorial library of cell-permeable bicyclic peptides. ACS Comb Sci 18: 7585.

Winter JJ, Anderson M, Blades K, Brassington C, Breeze AL, Chresta C, Embrey K, Fairley G, Faulder P, Finlay MR, et al. 2015. Small molecule binding sites on the Ras:SOS complex can be exploited for inhibition of Ras activation. J Med Chem 58: 2265-2274.

Wright LP, Philips MR. 2006. Thematic review series: Lipid posttranslational modifications. CAAX modification and membrane targeting of Ras. J Lipid Res 47: 883891.

Wu X, Upadhyaya P, Villalona-Calero MA, Briesewitz R, Pei D. 2013. Inhibition of ras-effector interaction by cyclic peptides. Med Chem Commun 4: 378-382.

Yang W, Soares J, Greninger P, Edelman EJ, Lightfoot H, Forbes S, Bindal N, Beare D, Smith JA, Thompson IR, et al. 2013. Genomics of drug sensitivity in cancer (GDSC): A resource for therapeutic biomarker discovery in cancer cells. Nucleic Acids Res 41: D955-D961.

Zhou Y, Hancock JF. 2015. Ras nanoclusters: Versatile lipidbased signaling platforms. Biochim Biophys Acta 1853: 841-849.

Zimmermann G, Papke B, Ismail S, Vartak N, Chandra A, Hoffmann M, Hahn SA, Triola G, Wittinghofer A, Bastiaens PI, et al. 2013. Small molecule inhibition of the KRAS-PDE $\delta$ interaction impairs oncogenic KRAS signalling. Nature 497: 638-642. 


\title{
$\&_{\mathrm{CSH}}^{\infty} \&$ Cold Spring Harbor

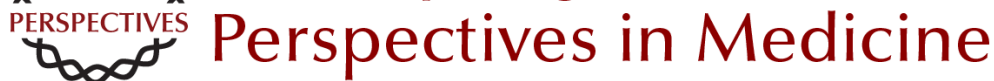

\section{Efforts to Develop KRAS Inhibitors}

\author{
Matthew Holderfield
}

Cold Spring Harb Perspect Med 2018; doi: 10.1101/cshperspect.a031864 originally published online November 3, 2017

\section{Subject Collection Ras and Cancer in the 21st Century}

Targeting Ras with Macromolecules Dehua Pei, Kuangyu Chen and Hui Liao

Ras-Specific GTPase-Activating Proteins-Structures, Mechanisms, and Interactions Klaus Scheffzek and Giridhar Shivalingaiah

Ras-Mediated Activation of the Raf Family Kinases Elizabeth M. Terrell and Deborah K. Morrison

Posttranslational Modifications of RAS Proteins Ian Ahearn, Mo Zhou and Mark R. Philips

Kras in Organoids Derek Cheng and David Tuveson

KRAS: The Critical Driver and Therapeutic Target for Pancreatic Cancer Andrew M. Waters and Channing J. Der

The K-Ras, N-Ras, and H-Ras Isoforms: Unique Conformational Preferences and Implications for Targeting Oncogenic Mutants Jillian A. Parker and Carla Mattos

PI3K: A Crucial Piece in the RAS Signaling Puzzle Agata Adelajda Krygowska and Esther Castellano
MRAS: A Close but Understudied Member of the RAS Family Lucy C. Young and Pablo Rodriguez-Viciana

The Interdependent Activation of

Son-of-Sevenless and Ras Pradeep Bandaru, Yasushi Kondo and John Kuriyan

Targeting the MAPK Pathway in RAS Mutant Cancers

Sarah G. Hymowitz and Shiva Malek

Ras and the Plasma Membrane: A Complicated Relationship Yong Zhou, Priyanka Prakash, Alemayehu A. Gorfe, et al.

Kras and Tumor Immunity: Friend or Foe? Jane Cullis, Shipra Das and Dafna Bar-Sagi

Synthetic Lethal Vulnerabilities in KRAS-Mutant Cancers Andrew J. Aguirre and William C. Hahn

Efforts to Develop KRAS Inhibitors Matthew Holderfield

Genetically Engineered Mouse Models of K-Ras-Driven Lung and Pancreatic Tumors: Validation of Therapeutic Targets Matthias Drosten, Carmen Guerra and Mariano Barbacid

For additional articles in this collection, see http://perspectivesinmedicine.cshlp.org/cgi/collection/ 